\title{
COMMON FIXED POINT THEOREMS IN MENGER SPACES
}

\author{
CHI-MING CHEN AND TONG-HUEI CHANG
}

Received 27 July 2005; Revised 3 May 2006; Accepted 7 May 2006

We proved two common fixed point theorems for four self-mappings and two set-valued mappings with $\phi$-contractive condition in a Menger space.

Copyright (c) 2006 Hindawi Publishing Corporation. All rights reserved.

\section{Introduction and preliminaries}

Probabilistic metric space was first introduced by Menger [6]. Later, there are many authors who have some detailed discussions and applications of a probabilistic metric space, for example, we may see Schweizer and Sklar [8]. Besides, there are many results about fixed point theorems in a probabilistic metric space with contractive types having appeared; we may see the papers [1-3, 9-12].

In this paper, we will prove two common fixed point theorems for four self-mappings and two set-valued mappings with $\phi$-contractive condition in a Menger space, which generalize some results of Dedeić and Sarapa [4, 5], and Sehgal and Bharucha-Reid [9].

A mapping $F: \mathbb{R} \rightarrow \mathbb{R}^{+}$is said to be a distribution if it is nondecreasing left continuous with $\inf \{F(t): t \in \mathbb{R}\}=0$ and $\sup \{F(t): t \in \mathbb{R}\}=1$.

We will denote by $\mathscr{L}$ the set of all distribution functions while $G$ will always denote the specific distribution function defined by

$$
G(t)= \begin{cases}0, & t \leq 0, \\ 1, & t>0 .\end{cases}
$$

A probabilistic metric space (PM-space) [7] is an ordered pair $\left(X, \mathscr{F}_{\mathscr{F}}\right)$ consisting of a nonempty set $X$ and a mapping $\mathscr{F}$ from $X \times X$ into the collections of all distribution functions on $\mathbb{R}$. For $x, y \in X$, we denote the distribution function $\mathscr{F}(x, y)$ by $F_{x, y}$ and $F_{x, y}(u)$ represents the value of $\mathscr{F}(x, y)$ at $u \in \mathbb{R}$. The functions $F_{x, y}$ are assumed to satisfy the following conditions:

(1) $F_{x, y}(u)=1$ for all $u>0$ if and only if $x=y$,

(2) $F_{x, y}(0)=0$ for all $x, y$ in $X$, 
2 Common fixed point theorems in Menger spaces

(3) $F_{x, y}(u)=F_{y, x}(u)$ for all $x, y$ in $X$, and

(4) if $F_{x, y}(u)=1$ and $F_{y, z}(v)=1$, then $F_{x, z}(u+v)=1$ for all $x, y, z$ in $X$ and $u, v>0$.

A mapping $t:[0,1] \times[0,1] \rightarrow[0,1]$ is called a $t$-norm if

(1) $t(a, 1)=a, t(0,0)=0$,

(2) $t(a, b)=t(b, a)$,

(3) $t(c, d) \geq t(a, b)$ for $c \geq a, d \geq b$, and

(4) $t(t(a, b), c)=t(a, t(b, c))$.

A Menger space is a triplet $(X, \mathscr{F}, t)$, where $(X, \mathscr{F})$ is a PM-space, $t$ is a $T$-norm, and the generalized triangle inequality

$$
F_{x, y}(u+v) \geq t\left(F_{x, y}(u), F_{y, z}(v)\right)
$$

holds for all $x, y, z$ in $X$ and $u, v>0$.

The concept of neighborhoods in a Menger space was introduced by Schweizer and Sklar [8].

Let $(X, \mathscr{F}, t)$ be a Menger space. If $x \in X, \varepsilon>0$, and $\lambda \in(0,1)$, then an $(\varepsilon, \lambda)$-neighborhood of $x$, called $U_{x}(\varepsilon, \lambda)$, is defined by

$$
U_{x}(\varepsilon, \lambda)=\left\{y \in X: F_{x, y}(\varepsilon)>1-\lambda\right\}
$$

An $(\varepsilon, \lambda)$-topology in $X$ is the topology induced by the family $\left\{U_{x}(\varepsilon, \lambda): x \in X, \varepsilon>0, \lambda \in\right.$ $(0,1)\}$ of neighborhood.

Remark 1.1. If $t$ is continuous, then Menger space $(X, \mathscr{F}, t)$ is a Hausdorff space in the $(\varepsilon, \lambda)$-topology. (see $[8])$.

Let $(X, \mathscr{F}, t)$ be a complete Menger space and $A \subset X$. Then $A$ is called a bounded set if

$$
\lim _{u \rightarrow \infty} \inf _{x, y \in A} F_{x, y}(u)=1
$$

Throughout this paper, $B(X)$ will denote the family of nonempty bounded subsets of a complete Menger space $X$.

For all $A, B \in B(X)$ and for all $u>0$, we define

$$
\begin{gathered}
{ }_{\delta} F_{A, B}(u)=\inf \left\{F_{x, y}(u): x \in A, y \in B\right\}, \\
{ }_{D} F_{A, B}(u)=\sup \left\{F_{x, y}(u): x \in A, y \in B\right\}, \\
{ }_{H} F_{A, B}(u)=\inf \left\{\sup _{a \in A} \inf F_{a, b}(u), \sup _{b \in B} \inf F_{a \in A}(u)\right\} .
\end{gathered}
$$

Remark 1.2. It is clear that ${ }_{\delta} F_{A, B}(u)={ }_{\delta} F_{B, A}(u),{ }_{D} F_{A, B}(u)={ }_{D} F_{B, A}(u)$, and ${ }_{H} F_{A, B}(u)=$ ${ }_{H} F_{B, A}(u)$, for all $A, B \in B(X)$ and $u>0$. 
If $A=\{x\}$, we denote ${ }_{\delta} F_{\{x\}, B}(u)={ }_{\delta} F_{x, B}(u),{ }_{D} F_{\{x\}, B}(u)={ }_{D} F_{x, B}(u)$, and ${ }_{H} F_{\{x\}, B}(u)=$ ${ }_{H} F_{x, B}(u)$.

Let $(X, \mathscr{F}, t)$ be a complete Menger space, and let $T: X \rightarrow B(X)$ be a set-valued function and $I: X \rightarrow X$ a single-valued function. Then we say that $S$ and $I$ are compatible if

$$
\lim _{n \rightarrow \infty} F_{S I x_{n}, I S x_{n}}(u)=1 \text {, }
$$

whenever $\left\{x_{n}\right\}$ is a sequence in $X$ such that

$$
\lim _{n \rightarrow \infty} \delta F_{I x_{n}, S x_{n}}(u)=1, \quad \forall u>0 .
$$

Let $\left\{A_{n}\right\}$ be a sequence in $B(X)$. We say that $\left\{A_{n}\right\} \delta$-converges to a set $A$ in $X$ if

$$
\lim _{n \rightarrow \infty}{ }_{\delta} F_{A_{n}, A}(u)=1, \quad \text { for every } u>0,
$$

and it is denoted by $A_{n} \stackrel{\delta}{\rightarrow} A$.

\section{Main results}

In this paper, we let $\mathbb{R}^{+}$denote the set of all nonnegative real numbers, let $\mathbb{N}$ denote the set of all positive integers, and let $(X, \mathscr{F}, t)$ be a Menger space with $t(x, y)=\min (x, y)$.

We first prove the following lemmas.

Lemma 2.1. Let $(X, \mathscr{F}, \mathrm{min})$ be a Menger space. Then for $A, B, C \in B(X)$ and for $u, v>0$,

$$
{ }_{\delta} F_{A, C}(u+v) \geq \min \left\{{ }_{\delta} F_{A, B}(u){ }_{,} F_{B, C}(v)\right\} .
$$

Proof. For all $u, v>0$, we have

$$
\min \left\{{ }_{\delta} F_{A, B}(u),{ }_{\delta} F_{B, C}(v)\right\} \leq \min \left\{F_{a, b}(u), F_{b, c}(v)\right\} \leq F_{a, c}(u+v)
$$

for each $a \in A, b \in B$, and $c \in C$.

This implies that $\min \left\{{ }_{\delta} F_{A, B}(u){ }_{, \delta} F_{B, C}(v)\right\} \leq{ }_{\delta} F_{A, C}(u+v)$.

Lemma 2.2. Let $(X, \mathscr{F}, \mathrm{min})$ be a Menger space. Then for $A, B \in B(X), c \in X$, and for $u, v>0$,

$$
{ }_{H} F_{A, c}(u+v) \geq \min \left\{{ }_{H} F_{A, B}(u),{ }_{H} F_{B, c}(v)\right\}
$$

Proof. Since for each $a, b, c \in X$ and for all $u, v>0$,

$$
F_{a, c}(u+v) \geq \min \left\{F_{a, b}(u), F_{b, c}(v)\right\} .
$$

By taking inf $f_{c \in C}$, we have

$$
\inf _{c \in C} F_{a, c}(u+v) \geq \min \left\{F_{a, b}(u), \inf _{c \in C} F_{b, c}(v)\right\} .
$$




\section{Common fixed point theorems in Menger spaces}

Hence,

$$
\begin{aligned}
\sup _{a \in A} \inf F_{a, c}(u+v) & \geq \sup _{a \in A} \min \left\{F_{a, b}(u), \inf _{c \in C} F_{b, c}(v)\right\} \\
& =\min \left\{\sup _{a \in A} F_{a, b}(u), \inf _{c \in C} F_{b, c}(v)\right\} \\
& \geq \min \left\{\sup _{a \in A} \inf _{b \in B} F_{a, b}(u), \inf _{c \in C} F_{b, c}(v)\right\} .
\end{aligned}
$$

Next, by taking $\sup _{b \in B}$, we have

$$
\begin{aligned}
\sup _{a \in A} \inf F_{a, c}(u+v) & \geq \sup _{b \in B} \min \left\{\sup _{a \in A} \inf _{b \in B} F_{a, b}(u), \inf _{c \in C} F_{b, c}(v)\right\} \\
& \geq \min \left\{\sup _{a \in A} \inf _{b \in B} F_{a, b}(u), \sup _{b \in B} \inf (v)\right\} .
\end{aligned}
$$

Similarly, for each $a, b, c \in X$ and for all $u, v>o$,

$$
F_{a, c}(u+v) \geq \min \left\{F_{a, b}(u), F_{b, c}(v)\right\}
$$

By taking $\inf _{c \in C}$, we have

$$
\inf _{a \in A} F_{a, c}(u+v) \geq \min \left\{\inf _{a \in A} F_{a, b}(u), F_{b, c}(v)\right\} .
$$

Hence,

$$
\begin{aligned}
\sup _{c \in C} \inf _{a \in A} F_{a, c}(u+v) & \geq \sup _{c \in C} \min \left\{\inf _{a \in A} F_{a, b}(u), F_{b, c}(v)\right\} \\
& =\min \left\{\inf _{a \in A} F_{a, b}(u), \sup _{c \in C} F_{b, c}(v)\right\} \\
& \geq \min \left\{\inf _{a \in A} F_{a, b}(u), \sup _{c \in C} \inf _{b \in B} F_{b, c}(v)\right\} .
\end{aligned}
$$

Next, by taking $\sup _{b \in B}$, we have

$$
\begin{aligned}
\sup _{c \in C} \inf _{a \in A} F_{a, c}(u+v) & \geq \sup _{b \in B} \min \left\{\inf _{a \in A} F_{a, b}(u), \sup _{c \in C} \inf F_{b, B}(v)\right\} \\
& \geq \min \left\{\sup _{b \in B} \inf _{a \in A} F_{a, b}(u), \sup _{c \in C} \inf (v)\right\} .
\end{aligned}
$$


Therefore, we obtain that

$$
\begin{aligned}
{ }_{H} F_{A, c}(u+v) & =\min \left\{\sup _{c \in C} \inf _{a \in A} F_{a, c}(u+v), \sup _{a \in A} \inf F_{a, c}(u+v)\right\} \\
& \geq \min \left\{\sup _{b \in B} \inf _{a \in A} F_{a, b}(u), \sup _{c \in C} \inf _{b \in B}(v), \sup _{a \in A} \inf F_{a, b}(u), \sup _{b \in B} \inf (v)\right\} \\
& =\min \left\{{ }_{c} F_{A, B}(u),{ }_{H} F_{B, c}(v)\right\} .
\end{aligned}
$$

Lemma 2.3. Let $(X, \mathscr{F}, \min )$ be a Menger space. If $A, B \in B(X)$, then $\lim _{u \rightarrow \infty} F_{A, B}(u)=1$.

Proof. For any $x \in A$ and $y \in B$, by Lemma 2.1, we have

$$
{ }_{\delta} F_{A, B}(u) \geq \min \left\{{ }_{\delta} F_{A, x}\left(\frac{u}{3}\right){ }_{\delta} F_{x, y}\left(\frac{u}{3}\right){ }_{\delta} F_{y, B}\left(\frac{u}{3}\right)\right\} .
$$

Letting $u \rightarrow \infty$, we have

$$
\lim _{u \rightarrow \infty} \delta F_{A, B}(u) \geq \min \left\{\lim _{u \rightarrow \infty} \delta F_{A, x}\left(\frac{u}{3}\right), \lim _{u \rightarrow \infty} \delta F_{x, y}\left(\frac{u}{3}\right), \lim _{u \rightarrow \infty} \delta F_{y, B}\left(\frac{u}{3}\right)\right\} .
$$

Since $x \in A, y \in B$, and $A, B \in B(X)$, we have

$$
\lim _{u \rightarrow \infty} F_{A, x}\left(\frac{u}{3}\right)=1
$$

Similarly, we have

$$
\lim _{u \rightarrow \infty} F_{y, B}\left(\frac{u}{3}\right)=1
$$

By the definition of the PM-space, we have that $\lim _{u \rightarrow \infty} F_{x, y}(u / 3)=1$.

Therefore, we conclude that

$$
\lim _{u \rightarrow \infty} F_{A, B}(u)=1
$$

This completes the proof.

The following lemma which was introduced by Chang [3], will play an important role for this paper.

LEMma 2.4. If $\phi: \mathbb{R}^{+} \rightarrow \mathbb{R}^{+}$is a strictly increasing, continuous function such that $0<\phi(u)<$ $u$ for all $u>0, \lim _{u \rightarrow \infty} \phi(u)=\infty$, and iffor each $u>0, \phi^{0}(u)=u$ and $\phi^{-n}(u)=\phi^{-1}\left(\phi^{-n+1}(u)\right)$ for each $n \in \mathbb{N}$ are denoted, then $\lim _{n \rightarrow \infty} \phi^{-n}(u)=\infty$.

In the sequel, we let $\Phi=\left\{\phi: \mathbb{R}^{+} \rightarrow \mathbb{R}^{+}: \phi\right.$ is a strictly increasing, continuous function with $\phi(t)<t$ for all $t>0\}$.

Lemma 2.5. Let $\left(X, \mathscr{F}_{F}, \mathrm{~min}\right)$ be a Menger space and $\left\{Y_{n}\right\}$ a sequence in $B(X)$. If for each $u>0$ and for each $n \in \mathbb{N}$,

$$
{ }_{\delta} F_{Y_{n+1}, Y_{n+2}}(\phi(u)) \geq{ }_{\delta} F_{Y_{n}, Y_{n+1}}(u), \quad \phi \in \Phi,
$$


6 Common fixed point theorems in Menger spaces

then

$$
\lim _{n \rightarrow \infty} F_{Y_{n}, Y_{n+1}}(u)=1 .
$$

Proof. For $u>0$, by induction, we have

$$
{ }_{\delta} F_{Y_{n+1}, Y_{n+2}}(u) \geq{ }_{\delta} F_{Y_{n}, Y_{n+1}}\left(\phi^{-1}(u)\right) \geq \cdots \geq{ }_{\delta} F_{Y_{1}, Y_{2}}\left(\phi^{-n}(u)\right), \quad \text { for each } n \in \mathbb{N} \text {. }
$$

By Lemma 2.4, we also have that $\phi^{-n}(u) \rightarrow \infty$, as $n \rightarrow \infty$.

Next, since $Y_{n}$ is a bounded set and ${ }_{\delta} F_{Y_{1}, Y_{2}}\left(\phi^{-n}(u)\right) \rightarrow 1$ as $n \rightarrow \infty$, hence we have

$$
\lim _{n \rightarrow \infty} F_{Y_{n+1}, Y_{n+2}}(u)=1 .
$$

Lemma 2.6. Let $(X, \mathscr{F}, \mathrm{min})$ be a Menger space, and let $A, B \in B(X)$. If

$$
{ }_{\delta} F_{A, B}(\phi(u)) \geq_{\delta} F_{A, B}(u), \quad \text { for } u>0,
$$

then $A=B=a$, for some $a \in X$.

Proof. For $u>0$, by induction, we have

$$
{ }_{\delta} F_{A, B}(u) \geq{ }_{\delta} F_{A, B}\left(\phi^{-1}(u)\right) \geq \cdots \geq{ }_{\delta} F_{A, B}\left(\phi^{-n}(u)\right) .
$$

Since $A, B \in B(X)$, by Lemma 2.3 , we have

$$
\lim _{n \rightarrow \infty} F_{A, B}\left(\phi^{-n}(u)\right)=1,
$$

and by Lemma 2.5, we have ${ }_{\delta} F_{A, B}(u)=1$ for $u>0$. Thus we conclude that $A=B=\{a\}$ for some $a \in X$.

The following lemma was introduced by Schweizer and Sklar [8].

Lemma 2.7. Let $(X, \mathscr{F}, \mathrm{min})$ be a Menger space. If $a_{n} \rightarrow a$ and $b_{n} \rightarrow b$, then for $u>0$,

$$
\lim _{n \rightarrow \infty} \inf F_{a_{n}, b_{n}}(u)=F_{a, b}(u) .
$$

From Lemma 2.7, we conclude the following lemma.

Lemma 2.8. Let $(X, \mathscr{F}, \min )$ be a Menger space. If $A_{n} \stackrel{\delta}{\rightarrow}$ a and $B_{n} \stackrel{\delta}{\rightarrow} b$, then for $u>0$,

$$
\lim _{n \rightarrow \infty} \inf _{\delta} F_{A_{n}, B_{n}}(u)=F_{a, b}(u)
$$

Proof. For $u>0$ and for $\varepsilon>0$. Since $F_{a, b}(u)$ is left continuous function at $u$, there exists a positive number $k$ with $0<2 k<u$ such that $F_{a, b}(u)-F_{a, b}(u-2 k)<\varepsilon$.

Since $k>0$ and $A_{n} \stackrel{\delta}{\rightarrow} a, B_{n} \stackrel{\delta}{\rightarrow} b$, hence we may take $m \in \mathbb{N}$ such that for $n \geq m$,

$$
{ }_{\delta} F_{A_{n}, a}(k) \geq F_{a, b}(u-2 k), \quad{ }_{\delta} F_{B_{n}, b}(k) \geq F_{a, b}(u-2 k) .
$$


Hence, for $n>m$,

$$
\begin{aligned}
{ }_{\delta} F_{A_{n}, B_{n}}(u) & \geq \min \left\{{ }_{\delta} F_{A_{n}, b}(u-k),{ }_{\delta} F_{b, B_{n}}(k)\right\} \\
& \geq \min \left\{F_{\delta} F_{A_{n}, a}(k),{ }_{\delta} F_{a, b}(u-2 k),{ }_{\delta} F_{b, B_{n}}(k)\right\}=F_{a, b}(u-2 k),
\end{aligned}
$$

and hence

$$
{ }_{\delta} F_{A_{n}, B_{n}}(u) \leq-F_{a, b}(u-2 k) .
$$

Therefore, we conclude that

$$
F_{a, b}(u)-{ }_{\delta} F_{A_{n}, B_{n}}(u)<F_{a, b}(u)-F_{a, b}(u-2 k)<\varepsilon .
$$

Taking $\lim _{n \rightarrow \infty}$ inf, we have

$$
F_{a, b}(u)-\lim _{n \rightarrow \infty} \inf _{\delta} F_{A_{n}, B_{n}}(u)<\varepsilon .
$$

For any $a_{n} \in A_{n}, b_{n} \in B_{n}$, since $A_{n} \stackrel{\delta}{\rightarrow} a$ and $B_{n} \stackrel{\delta}{\rightarrow} b$, we have $a_{n} \rightarrow a, b_{n} \rightarrow b$. Thus, for $u>0$

$$
{ }_{\delta} F_{A_{n}, B_{n}}(u) \leq F_{a_{n}, b_{n}}(u)
$$

Taking $\lim _{n \rightarrow \infty}$ inf, we have

$$
\lim _{n \rightarrow \infty} \inf _{\delta} F_{A_{n}, B_{n}}(u) \leq \lim _{n \rightarrow \infty} \inf F_{a_{n}, b_{n}}(u) .
$$

By Lemma 2.7, we have

$$
\lim _{n \rightarrow \infty} \inf F_{a_{n}, b_{n}}(u)=F_{a, b}(u), \text { and so } F_{a, b}(u)-\lim _{n \rightarrow \infty} \inf _{\delta} F_{A_{n}, B_{n}}(u) \geq 0 \text {. }
$$

Therefore, for any $\varepsilon>0$,

$$
\varepsilon>F_{a, b}(u)-\lim _{n \rightarrow \infty} \inf _{\delta} F_{A_{n}, B_{n}}(u) \geq 0 .
$$

This implies that

$$
\liminf _{n \rightarrow \infty} F_{A_{n}, B_{n}}(u)=F_{a, b}(u), \quad \text { for } u>0 .
$$

The following two theorems are our main results for this paper. 
Theorem 2.9. Let $(X, \mathscr{F}, \mathrm{min})$ be a complete Menger space. Let $f, g, \eta, \xi: X \rightarrow X$ be four single-valued functions, and let $S, T: X \rightarrow B(X)$ two set-valued functions. If the following conditions are satisfied:

(i) $S(X) \subset \xi g(X), T(X) \subset \eta f(X)$,

(ii) $\eta f=f \eta, \xi g=g \xi, S f=f S, T g=g T$,

(iii) $\eta f$ or $\xi g$ is continuous,

(iv) $(S, \eta f)$ and $(T, \xi g)$ are compatible, and

(v) for $u>0$,

$$
\begin{aligned}
& { }_{\delta} F_{S x, T y}(\phi(u)) \\
& \quad \geq \min \left\{F_{\eta f x, \xi g y}(u),{ }_{\delta} F_{\eta f x, S x}(u),{ }_{\delta} F_{\xi g y, T y}(u),{ }_{\delta} F_{\xi g y, S x}(\beta u),{ }_{\delta} F_{\eta f x, T y}((2-\beta) u)\right\}
\end{aligned}
$$

for all $x, y \in X, \beta \in(0,2)$, where $\phi \in \Phi$, then $f, g, \eta, \xi, S$, and $T$ have a unique common fixed point $z$ in $X$.

Proof. Let $x_{0} \in X$. Define the sequence $\left\{x_{n}\right\}$ recursively as follows:

$$
\xi g x_{2 n+1} \in S x_{2 n}=Z_{2 n}, \quad \eta f x_{2 n+2} \in T x_{2 n+1}=Z_{2 n+1} .
$$

For $n \in \mathbb{N}$ and for all $u>0$, and $\beta=(1-\alpha)$ with $\alpha \in(0,1)$,

$$
\begin{aligned}
{ }_{\delta} F_{Z_{2 n}, Z_{2 n+1}}(\phi(u)) \\
={ }_{\delta} F_{S x_{2 n}, T x_{2 n+1}}(\phi(u)) \\
\geq \min \left\{F_{\eta f x_{2 n}, \xi g x_{2 n+1}}(u),{ }_{\delta} F_{\eta f x_{2 n}, S x_{2 n}}(u),{ }_{\delta} F_{\xi g x_{2 n+1}, T x_{2 n+1}}(u),{ }_{\delta} F_{\xi g x_{2 n+1}, S x_{2 n}}((1-\alpha) u),\right. \\
\\
\left.\quad{ }_{\delta} F_{\eta f x_{2 n}, T x_{2 n+1}}((1+\alpha) u)\right\} \\
\geq \min \left\{{ }_{\delta} F_{Z_{2 n-1}, Z_{2 n}}(u),{ }_{\delta} F_{Z_{2 n-1}, Z_{2 n}}(u),{ }_{\delta} F_{Z_{2 n}, Z_{2 n+1}}(u),{ }_{\delta} F_{Z_{2 n}, Z_{2 n}}((1-\alpha) u),\right. \\
\left.\quad{ }_{\delta} F_{Z_{2 n-1}, Z_{2 n+1}}((1+\alpha) u)\right\} \\
\geq \min \left\{{ }_{\delta} F_{Z_{2 n-1}, Z_{2 n}}(u),{ }_{\delta} F_{Z_{2 n-1}, Z_{2 n}}(u),{ }_{\delta} F_{Z_{2 n}, Z_{2 n+1}}(u), 1,{ }_{\delta} F_{Z_{2 n-1}, Z_{2 n}}\left((u),{ }_{\delta} F_{Z_{2 n}, Z_{2 n+1}}(\alpha u)\right)\right\} \\
=\min \left\{{ }_{\delta} F_{Z_{2 n-1}, Z_{2 n}}(u),{ }_{\delta} F_{Z_{2 n}, Z_{2 n+1}}(u),{ }_{\delta} F_{Z_{2 n}, Z_{2 n+1}}(\alpha u)\right\} .
\end{aligned}
$$

As $t$-norm $=\min$ is continuous, letting $\alpha \rightarrow 1$, we have

$$
{ }_{\delta} F_{Z_{2 n}, Z_{2 n+1}}(\phi(u)) \geq \min \left\{{ }_{\delta} F_{Z_{2 n-1}, Z_{2 n}}(u),{ }_{\delta} F_{Z_{2 n}, Z_{2 n+1}}(u)\right\} .
$$


By Lemma 2.6, we have

$$
{ }_{\delta} F_{Z_{2 n}, Z_{2 n+1}}(\phi(u)) \geq_{\delta} F_{Z_{2 n-1}, Z_{2 n}}(u)
$$

Similarly, we also can prove that for $n \in \mathbb{N}$ and for all $u>0$,

$$
{ }_{\delta} F_{Z_{2 n+1}, Z_{2 n+2}}(\phi(u)) \geq{ }_{\delta} F_{Z_{2 n}, Z_{2 n+1}}(u) .
$$

So, we have

$$
{ }_{\delta} F_{Z_{n+1}, Z_{n+2}}(\phi(u)) \geq{ }_{\delta} F_{Z_{n}, Z_{n+1}}(u), \quad \forall n \in \mathbb{N}, u>0 .
$$

By Lemma 2.5, we conclude that

$$
\lim _{n \rightarrow \infty} \delta F_{Z_{n}, Z_{n+1}}(u)=1, \quad \forall u>0
$$

Now, we consider the condition $(v)$ with $\beta=1$, and then we claim that

for $\varepsilon>0, \lambda \in(0,1) \quad$ there is $M(\varepsilon, \lambda) \in \mathbb{N}$ such that ${ }_{\delta} F_{Z_{n}, Z_{m}}(\varepsilon) \geq 1-\lambda$ for $n, m \geq M$.

If it is not the case, then there exists $\varepsilon^{\prime}>0, \lambda^{\prime} \in(0,1)$ such that for $k \in \mathbb{N}$, there exist $n_{k}>m_{k} \geq k$ such that

(1) $n_{k}$ is even and $m_{k}$ is odd,

(2) ${ }_{\delta} F_{Z_{n_{k}}, Z_{m_{k}}}\left(\varepsilon^{\prime}\right)<1-\lambda^{\prime}$, and

(3) $n_{k}$ is the smallest even number such that (1) and (2) hold.

By $(*)$, we may choose $m_{1} \in \mathbb{N}$ such that for $n \geq m_{1}$,

$$
{ }_{\delta} F_{Z_{n}, Z_{n+1}}\left(\min \left\{\frac{\varepsilon^{\prime}}{2}, \frac{\phi^{-1}\left(\varepsilon^{\prime}\right)-\varepsilon^{\prime}}{2}\right\}\right)>1-\lambda^{\prime} .
$$

So for $k>m_{1}, n_{k} \geq m_{k}+3$, and so for $k>m_{1}$,

$$
\begin{aligned}
& 1-\lambda^{\prime}>_{\delta} F_{Z_{n_{k}}, Z_{m_{k}}}\left(\varepsilon^{\prime}\right)={ }_{\delta} F_{S x_{n_{k}}, T x_{m_{k}}}\left(\varepsilon^{\prime}\right) \\
& \geq \min \left\{F_{\eta f_{x_{n_{k}}}, \xi g_{x_{m_{k}}}}\left(\phi^{-1}\left(\varepsilon^{\prime}\right)\right),{ }_{\delta} F_{\eta f_{x_{n_{k}}}, S_{x_{n_{k}}}}\left(\phi^{-1}\left(\varepsilon^{\prime}\right)\right),{ }_{\delta} F_{\xi g_{x_{m_{k}}}, T_{x_{m_{k}}}}\left(\phi^{-1}\left(\varepsilon^{\prime}\right)\right),\right. \\
& \left.{ }_{\delta} F_{\xi_{x_{m_{k}}}, S_{x_{n_{k}}}}\left(\phi^{-1}\left(\varepsilon^{\prime}\right)\right),{ }_{\delta} F_{\eta f_{x_{n_{k}}}, T_{x_{m_{k}}}}\left(\phi^{-1}\left(\varepsilon^{\prime}\right)\right)\right\} \\
& \geq \min \left\{{ }_{\delta} F_{Z_{n_{k-1}}, Z_{m_{k-1}}}\left(\phi^{-1}\left(\varepsilon^{\prime}\right)\right),{ }_{\delta} F_{Z_{n_{k-1}}, Z_{n_{k}}}\left(\phi^{-1}\left(\varepsilon^{\prime}\right)\right),{ }_{\delta} F_{Z_{m_{k-1}}, Z_{m_{k}}}\left(\phi^{-1}\left(\varepsilon^{\prime}\right)\right)\right. \text {, } \\
& \left.{ }_{\delta} F_{Z_{n_{k}}, Z_{m_{k-1}}}\left(\phi^{-1}\left(\varepsilon^{\prime}\right)\right),{ }_{\delta} F_{Z_{n_{k-1}}, Z_{m_{k}}}\left(\phi^{-1}\left(\varepsilon^{\prime}\right)\right)\right\} .
\end{aligned}
$$


Since

$$
\begin{aligned}
& { }_{\delta} F_{Z_{n_{k-1}}, Z_{m_{k}}}\left(\phi^{-1}\left(\varepsilon^{\prime}\right)\right) \geq \min \left\{{ }_{\delta} F_{Z_{n_{k-1}}, Z_{n_{k-2}}}\left(\phi^{-1}\left(\varepsilon^{\prime}\right)-\varepsilon^{\prime}\right),{ }_{\delta} F_{Z_{n_{k-2}}, Z_{m_{k}}}\left(\varepsilon^{\prime}\right)\right\}, \\
& { }_{\delta} F_{Z_{m_{k-1}}, Z_{n_{k}}}\left(\phi^{-1}\left(\varepsilon^{\prime}\right)\right) \geq \min \left\{{ }_{\delta} F_{Z_{m_{k-1}}, Z_{n_{k-1}}}\left(\frac{\phi^{-1}\left(\varepsilon^{\prime}\right)+\varepsilon^{\prime}}{2}\right),{ }_{\delta} F_{Z_{n_{k-1}}, Z_{n_{k}}}\left(\frac{\phi^{-1}\left(\varepsilon^{\prime}\right)-\varepsilon^{\prime}}{2}\right)\right\} \\
& \geq \min \left\{{ }_{\delta} F_{Z_{n_{k-1}}, Z_{n_{k-2}}}\left(\frac{\phi^{-1}\left(\varepsilon^{\prime}\right)-\varepsilon^{\prime}}{2}\right),{ }_{\delta} F_{Z_{n_{k-2}}, Z_{m_{k-1}}}\left(\varepsilon^{\prime}\right),\right. \\
& \left.{ }_{\delta} F_{Z_{n_{k-1}}, Z_{n_{k}}}\left(\frac{\phi^{-1}\left(\varepsilon^{\prime}\right)-\varepsilon^{\prime}}{2}\right)\right\} \\
& \geq \min \left\{{ }_{\delta} F_{Z_{n_{k-1}}, Z_{n_{k-2}}}\left(\frac{\phi^{-1}\left(\varepsilon^{\prime}\right)-\varepsilon^{\prime}}{2}\right),{ }_{\delta} F_{Z_{n_{k-2}}, Z_{m_{k}}}\left(\frac{\varepsilon^{\prime}}{2}\right),{ }_{\delta} F_{Z_{m_{k}}, Z_{m_{k-1}}}\left(\frac{\varepsilon^{\prime}}{2}\right),\right. \\
& \left.{ }_{\delta} F_{Z_{n_{k-1}}, Z_{n_{k}}}\left(\frac{\phi^{-1}\left(\varepsilon^{\prime}\right)-\varepsilon^{\prime}}{2}\right)\right\} \\
& \geq \min \left\{{ }_{\delta} F_{Z_{n_{k-1}}, Z_{n_{k-2}}}\left(\varepsilon^{\prime}\right),{ }_{\delta} F_{Z_{n_{k-2}}}, Z_{m_{k}}\left(\varepsilon^{\prime}\right),_{\delta} F_{Z_{m_{k}}, Z_{m_{k}}}\left(\frac{\phi^{-1}\left(\varepsilon^{\prime}\right)-\varepsilon^{\prime}}{2}\right)\right. \\
& \left.{ }_{\delta} F_{Z_{m_{k}}}, Z_{m_{k-1}}\left(\varepsilon^{\prime}\right), \delta F_{Z_{n_{k-1}}, Z_{n_{k}}}\left(\frac{\phi^{-1}\left(\varepsilon^{\prime}\right)-\varepsilon^{\prime}}{2}\right)\right\} \\
& { }_{\delta} F_{Z_{n_{k-1}}, Z_{m_{k-1}}}\left(\phi^{-1}\left(\varepsilon^{\prime}\right)\right) \geq \min \left\{{ }_{\delta} F_{Z_{n_{k-1}}, Z_{n_{k-2}}}\left(\frac{\phi^{-1}\left(\varepsilon^{\prime}\right)-\varepsilon^{\prime}}{2}\right),{ }_{\delta} F_{Z_{n_{k-2}}, Z_{m_{k-1}}}\left(\frac{\phi^{-1}\left(\varepsilon^{\prime}\right)+\varepsilon^{\prime}}{2}\right)\right\} \\
& \geq \min \left\{{ }_{\delta} F_{Z_{n_{k-1}}, Z_{n_{k-2}}}\left(\frac{\phi^{-1}\left(\varepsilon^{\prime}\right)-\varepsilon^{\prime}}{2}\right),{ }_{\delta} F_{Z_{n_{k-2}}, Z_{m_{k}}}\left(\varepsilon^{\prime}\right)\right. \\
& \left.{ }_{\delta} F_{Z_{m_{k-1}}, Z_{m_{k}}}\left(\frac{\phi^{-1}\left(\varepsilon^{\prime}\right)-\varepsilon^{\prime}}{2}\right)\right\}
\end{aligned}
$$

so for $k>m_{1}$, we have

$$
1-\lambda^{\prime}>_{\delta} F_{Z_{n_{k}}, Z_{m_{k}}}\left(\varepsilon^{\prime}\right) \geq 1-\lambda^{\prime}
$$

which is a contradiction. And, since $X$ is complete, hence for any choice of $z_{n}$ in $Z_{n}$, the sequence $\left\{z_{n}\right\}$ must converge to some point, say, $z$ in $X$. The point $z$ is independent of the choice of $z_{n}$ and so we have

$$
\eta f x_{2 n} \longrightarrow z, \quad \xi g x_{2 n+1} \longrightarrow z, \quad S x_{2 n} \longrightarrow\{z\}, \quad T x_{2 n+1} \longrightarrow\{z\}
$$

That is, for $u>0$,

$F_{\eta f x_{2 n}, z}(u) \longrightarrow 1, \quad F_{\xi g x_{2 n+1}, z}(u) \longrightarrow 1, \quad{ }_{\delta} F_{S x_{2 n}, z}(u) \longrightarrow 1, \quad{ }_{\delta} F_{T x_{2 n+1}, z}(u) \longrightarrow 1$ as $n \longrightarrow \infty$ 
Assume that the function $\eta f$ is continuous, then for $u>0$, we have

$$
\lim _{n \rightarrow \infty} F_{(\eta f)^{2} x_{2 n}, \eta f z}(u)=1, \quad \lim _{n \rightarrow \infty} \delta F_{\eta f S x_{2 n}, \eta f z}(u)=1 .
$$

By $\lim _{n \rightarrow \infty} F_{\eta f x_{2 n}, z}(u)=1$ and $\lim _{n \rightarrow \infty} F_{S x_{2 n}, z}(u)=1$, we obtain $\lim _{n \rightarrow \infty \delta} F_{S x_{2 n}, \eta f x_{2 n}}(u)=$ 1. Since $S$ and $\eta f$ are compatible, and for $u>0, \lim _{n \rightarrow \infty} F_{S x_{2 n}, \eta f x_{2 n}}(u)=1$, we have $\lim _{n \rightarrow \infty} F_{\eta f S x_{2 n}, S \eta f x_{2 n}}(u)=1$ and ${ }_{H} F_{S \eta f x_{2 n}, \eta f z}(u) \geq \min \left\{{ }_{H} F_{\eta f S x_{2 n}, S \eta f x_{2 n}}(u / 2),{ }_{H} F_{\eta f S x_{2 n}, \eta f z}(u /\right.$ 2)\}. And, since $\lim _{n \rightarrow \infty} F_{\eta f S x_{2 n}, S \eta f x_{2 n}}(u / 2)=1, \lim _{n \rightarrow \infty} F_{\eta f S x_{2 n}, \eta f z}(u / 2)=1$, we have

$$
\lim _{n \rightarrow \infty}{ }_{H} F_{S \eta f x_{2 n}, \eta f z}(u)=\lim _{n \rightarrow \infty} \delta F_{S \eta f x_{2 n}, \eta f z}(u)=1 .
$$

In order to complete the proof, we will divide it into 5 steps as follows:

Step 1. For $u>0$ with $\beta=1$ in the condition ( $v)$,

$$
\begin{aligned}
{ }_{\delta} F_{S \eta f x_{2 n}, T x_{2 n+1}}(\phi(u)) \geq \min \{ & F_{(\eta f)^{2} x_{2 n}, \xi g x_{2 n+1}}(u),{ }_{\delta} F_{(\eta f)^{2} x_{2 n}, S \eta f x_{2 n}}(u),{ }_{\delta} F_{\xi g x_{2 n+1}, T x_{2 n+1}}(u), \\
& \left.{ }_{\delta} F_{\xi g x_{2 n+1}, S \eta f x_{2 n}}(u),{ }_{\delta} F_{(\eta f)^{2} x_{2 n}, T x_{2 n+1}}(u)\right\} .
\end{aligned}
$$

Taking $\lim _{n \rightarrow \infty}$, by Lemma 2.8,

$$
F_{\eta f z, z}(\phi(u)) \geq \min \left\{F_{\eta f z, z}(u), F_{\eta f z, \eta f z}(u), F_{z, z}(u), F_{\eta f z, z}(u), F_{\eta f z, z}(u)\right\}=F_{\eta f z, z}(u) .
$$

So we get $\eta f z=z$.

Step 2. For $u>0$ with $\beta=1$ in the condition $(v)$,

$$
\begin{aligned}
& { }_{\delta} F_{S z, z}(\phi(u)) \\
& \quad=\lim _{n \rightarrow \infty} \inf _{\delta} F_{S z, T x_{2 n+1}}(\phi(u)) \\
& \quad \geq \lim _{n \rightarrow \infty} \inf \min \left\{F_{\eta f z, \xi g x_{2 n+1}}(u),{ }_{\delta} F_{\eta f z, S z}(u),{ }_{\delta} F_{\xi g x_{2 n+1}, T x_{2 n+1}}(u),{ }_{\delta} F_{S z, \xi g x_{2 n+1}}(u),_{\delta} F_{\eta f z, T x_{2 n+1}}(u)\right\} \\
& \quad \geq \min \left\{F_{z, z}(u),{ }_{\delta} F_{z, S z}(u), F_{z, z}(u),{ }_{\delta} F_{z, S z}(u), F_{z, z}(u)\right\}={ }_{\delta} F_{z, S z}(u) .
\end{aligned}
$$

So we get $S z=\{z\}$.

Hence, by Steps 1 and 2, we have $S z=\{z\}=\{\eta f z\}$.

Step 3. By the condition (i), since $S X \subset \xi g X$, there exists $z^{\prime} \in X$ such that $\left\{\xi g z^{\prime}\right\}=S z=$ $\{z\}$.

So for any $u>0$ with $\beta=1$ in the condition $(v)$

$$
\begin{aligned}
& { }_{\delta} F_{S x_{2 n}, T z^{\prime}}(\phi(u)) \\
& \quad \geq \min \left\{F_{\eta f x_{2 n}, \xi g z^{\prime}}(u),{ }_{\delta} F_{\eta f x_{2 n}, S x_{2 n}}(u),{ }_{\delta} F_{\xi g z^{\prime}, T z^{\prime}}(u),{ }_{\delta} F_{\eta f z^{\prime}, S x_{2 n}}(u),{ }_{\delta} F_{\eta f x_{2 n}, T z^{\prime}}(u)\right\} .
\end{aligned}
$$

Taking $\lim _{n \rightarrow \infty}$ inf, by Lemma 2.8,

$$
{ }_{\delta} F_{z, T z^{\prime}}(\phi(u)) \geq \min \left\{F_{z, z}(u), F_{z, z}(u),{ }_{\delta} F_{z, T z^{\prime}}(u), F_{z, z}(u),{ }_{\delta} F_{z, T z^{\prime}}(u)\right\}={ }_{\delta} F_{z, T z^{\prime}}(u) .
$$


So we get $T z^{\prime}=\{z\}$. Hence, $\left\{\xi g z^{\prime}\right\}=\{z\}=T z^{\prime}$.

By Step 2, we may let $\{z\}=\{\eta f z\}=\{S z\}=\left\{\xi g z^{\prime}\right\}=\left\{T z^{\prime}\right\}$.

Since $S$ and $\eta f$ are compatible and $\{\eta f z\}=S z$, we get $\eta f S z=S \eta f z$, that is, $\{\eta f z\}=S z$.

Now,

$$
\begin{aligned}
{ }_{\delta} F_{S z, z} & (\phi(u))={ }_{\delta} F_{S z, T z^{\prime}}(\phi(u)) \\
& \geq \min \left\{F_{\eta f z, \xi g z^{\prime}}(u),{ }_{\delta} F_{\eta f z, S z}(u),{ }_{\delta} F_{\xi g z^{\prime}, T z^{\prime}}(u),{ }_{\delta} F_{\eta f z, T z^{\prime}}(u),{ }_{\delta} F_{S z, \xi g z^{\prime}}(u)\right\} \\
& ={ }_{\delta} F_{\eta f z, z}(u)={ }_{\delta} F_{S z, z}(u) .
\end{aligned}
$$

This implies $S z=\{z\}=\{\eta f z\}$.

Choose $z^{\prime}$ in $X$ such that $\left\{\xi g z^{\prime}\right\}=S z=\{z\}$, then

$$
\begin{aligned}
{ }_{\delta} F_{z, T z^{\prime}} & (\phi(u)) \\
& ={ }_{\delta} F_{S z, T z^{\prime}}(\phi(u)) \\
& \geq \min \left\{F_{\eta f z, \xi g z^{\prime}},{ }_{\delta} F_{\eta f z, S z}(u),{ }_{\delta} F_{\xi g z^{\prime}, T z^{\prime}}(u){ }_{\delta} F_{\eta f z, T z^{\prime}}(u){ }_{\delta} F_{S z, \xi g z^{\prime}}(u)\right\}={ }_{\delta} F_{z, T z^{\prime}}(u) .
\end{aligned}
$$

By Lemma 2.6, we get $T z^{\prime}=\{z\}$.

Since $T$ and $\xi g$ are compatible and $\left\{\xi g z^{\prime}\right\}=T z^{\prime}$, we get $T \xi g z^{\prime}=\xi g T z^{\prime}$, that is, $T z=$ $\{\xi g z\}$.

Now, for $u>0$,

$$
\begin{aligned}
{ }_{\delta} F_{S z, T z} & (\phi(u)) \\
& \geq \min \left\{F_{\eta f z, \xi g z}(u){ }_{\delta} F_{\eta f z, S z}(u),{ }_{\delta} F_{\xi g z, T z}(u),{ }_{\delta} F_{\eta f z, T z}(u),{ }_{\delta} F_{S z, \xi g z}(u)\right\} \\
& =F_{\eta f z, \xi g z}(u)={ }_{\delta} F_{S z, T z}(u) .
\end{aligned}
$$

So we have $S z=T z=\{\eta f z\}=\{\xi g z\}=\{z\}$.

Step 4. For $u>0$ with $\beta=1$ in the condition $(v)$, we get

$$
\begin{aligned}
& { }_{\delta} F_{S f z, T x_{2 n+1}}(\phi(u)) \\
& \quad \geq \min \left\{F_{\eta f f z, \xi g x_{2 n+1}}(u),{ }_{\delta} F_{\eta f f z, S f z}(u),{ }_{\delta} F_{\xi g x_{2 n+1}, T x_{2 n+1}}(u),{ }_{\delta} F_{\xi g x_{2 n+1}, S f z}(u),{ }_{\delta} F_{\eta f f z, T x_{2 n+1}}(u)\right\} .
\end{aligned}
$$

By the condition (ii), $\eta f=f \eta, S f=f S$, so we have $\eta f(f z)=f(\eta f z)=f z$ and $S(f z)=$ $\{f(S z)\}=\{f z\}$. Taking $\lim _{n \rightarrow \infty}$ inf, by Lemma 2.8,

$$
F_{f z, z}(\phi(u)) \geq \min \left\{F_{f z, z}(u), F_{f z, f z}(u), F_{z, z}(u), F_{z, f z}(u), F_{f z, z}(u)\right\}=F_{f z, z}(u) .
$$

So we get $f z=z$.

Hence, by Steps 1 and 4, we have $\eta f z=z$ and $f z=z$, which implies $\eta z=z$. Therefore, $\{z\}=\{f z\}=\{\eta z\}=S z$. 
Step 5. For $u>0$ with $\beta=1$ in condition $(v)$, we get

$$
\begin{aligned}
& { }_{\delta} F_{S x_{2 n}, T g z}(\phi(u)) \\
& \quad \geq \min \left\{F_{\eta f x_{2 n}, \xi g g z}(u),{ }_{\delta} F_{\eta f x_{2 n}, S x_{2 n}}(u),{ }_{\delta} F_{\xi g g z, T g z}(u),{ }_{\delta} F_{\xi g g z, S x_{2 n}}(u),{ }_{\delta} F_{\eta f x_{2 n}, T g z}(u)\right\} .
\end{aligned}
$$

Since $T g=g T$ and $\xi g=g \xi$, we have $T g z=\{g T z\}=\{g z\}$ and $\xi g(g z)=g(\xi g z)=g z$. Taking $\lim _{n \rightarrow \infty}$ inf, by Lemma 2.8 , we get

$$
F_{z, g z}(\phi(u)) \geq \min \left\{F_{z, g z}(u), F_{z, z}(u), F_{g z, g z}(u), F_{g z, z}(u), F_{z, g z}(u)\right\}=F_{z, g z}(u) .
$$

So we get $g z=z$.

Hence, by Steps 3 and 5, we have $\xi g z=z$ and $g z=z$, which implies $\xi z=z$.

So we have $\{z\}=\{g z\}=\{\xi z\}=T z$.

Therefore, we have

$$
\{z\}=\{f z\}=\{g z\}=\{\eta z\}=\{\xi z\}=S z=T z .
$$

Last, we want to prove the uniqueness. Let $y$ be the another commom fixed point of $\eta$, $f, \xi, g, S$, and $T$. Then for $u>0$,

$$
\begin{aligned}
F_{z, y}(\phi(u)) & ={ }_{\delta} F_{S z, T y}(\phi(u)) \\
& \geq \min \left\{F_{\eta f z, \xi g y}(u),{ }_{\delta} F_{\eta f z, S z}(u){ }_{\delta} F_{\xi g y, T y}(u){ }_{,} F_{\xi g y, S z}(u){ }_{\delta} F_{\eta f z, T y}(u)\right\} \\
& \geq \min \left\{F_{z, y}(u), F_{z, z}(u), F_{y, y}(u), F_{y, z}(u), F_{y g z}(u)\right\}=F_{z, y}(u) .
\end{aligned}
$$

This implies $y=z$. We complete the proof.

If we take $f=g=I$, the identity map on $X$ in Theorem 2.9, then we immediately have the following corollary.

Corollary 2.10. Let $(X, F, \mathrm{~min})$ be a complete Menger space. Let $\eta, \xi: X \rightarrow X$ be two single-valued functions, and let $S, T: X \rightarrow B(X)$ be two set-valued functions. If the following conditions are satisfied:

(i) $S(X) \subset \xi(X), T(X) \subset \eta(X)$,

(ii) $\eta$ or $\xi$ is continuous,

(iii) $(S, \eta)$ and $(T, \xi)$ are compatible,

(iv) for $u>0$,

$$
{ }_{\delta} F_{S x, T y}(\phi(u)) \geq \min \left\{F_{\eta x, \xi y}(u),{ }_{\delta} F_{\eta x, S x}(u),{ }_{\delta} F_{\xi y, T y}(u),{ }_{\delta} F_{\xi y, S x}(\beta u),{ }_{\delta} F_{\eta x, T y}((2-\beta) u)\right\}
$$

for all $x, y \in X, \beta \in(0,2)$, where $\phi \in \Phi$, then $\eta, \xi, S$, and $T$ have a unique common fixed point $z$ in $X$.

By the same process of the proof of Theorem 2.9, we also get the results of Theorem 2.11. 
Theorem 2.11. Let $(X, \mathscr{F}, \mathrm{min})$ be a complete Menger space. Let $f, g, \eta, \xi: X \rightarrow X$ be four single-valued functions, and let $S, T: X \rightarrow B(X)$ be two set-valued functions. If the following conditions are satisfied:

(i) $S(X) \subset \xi g(X), T(X) \subset \eta f(X)$,

(ii) $\eta f=f \eta, \xi g=g \xi, S f=f S, T g=g T$,

(iii) $\eta f$ or $\xi g$ is continuous,

(iv) $(S, \eta f)$ and $(T, \xi g)$ are compatible,

(v) for $u>0$,

$$
{ }_{\delta} F_{S x, T y}(\phi(u)) \geq \min \left\{F_{\eta f x, \xi g y}(u),{ }_{\delta} F_{\eta f x, S x}(u),{ }_{\delta} F_{\xi g y, T y}(u),{ }_{D} F_{\xi g y, S x}(u)+{ }_{D} F_{\eta f x, T y}(u)\right\}
$$

for all $x, y \in X$, where $\phi \in \Phi$, then $f, g, \eta, \xi, S$, and $T$ have a unique common fixed point $z$ in $X$.

If we take $f=g=I$, the identity map on $X$ in Theorem 2.11, then we immediately have the following corollary.

Corollary 2.12. Let $(X, \mathscr{F}, \mathrm{min})$ be a complete Menger space. Let $\eta, \xi: X \rightarrow X$ be two single-valued functions, and let $S, T: X \rightarrow B(X)$ be two set-valued functions. If the following conditions are satisfied:

(i) $S(X) \subset \xi(X), T(X) \subset \eta(X)$,

(ii) $\eta$ or $\xi$ is continuous,

(iii) $(S, \eta)$ and $(T, \xi)$ are compatible,

(iv) for $u>0$,

$$
{ }_{\delta} F_{S x, T y}(\phi(u)) \geq \min \left\{F_{\eta x, \xi y}(u),{ }_{\delta} F_{\eta x, S x}(u),{ }_{\delta} F_{\xi y, T y}(u),{ }_{D} F_{\xi y, S x}(u)+_{D} F_{\eta x, T y}(u)\right\}
$$

for all $x, y \in X$, where $\phi \in \Phi$, then $\eta, \xi, S$, and T have a unique common fixed point $z$ in $X$.

\section{References}

[1] G. L. Cain Jr. and R. H. Kasriel, Fixed and periodic points of local contraction mappings on probabilistic metric spaces, Mathematical Systems Theory 9 (1975/1976), no. 4, 289-297.

[2] K. P. Chamola, Fixed points of mappings satisfying a new contraction condition in random normed spaces, Mathematica Japonica 33 (1988), no. 6, 821-825.

[3] T.-H. Chang, Common fixed point theorems in Menger spaces, Bulletin of the Institute of Mathematics. Academia Sinica 22 (1994), no. 1, 17-29.

[4] R. Dedeić and N. Sarapa, On common fixed point theorems for commuting mappings on Menger spaces, Radovi Matematički 4 (1988), no. 2, 269-278.

[5] A A common fixed point theorem for three mappings on Menger spaces, Mathematica Japonica 34 (1989), no. 6, 919-923.

[6] K. Menger, Statistical metrics, Proceedings of the National Academy of Sciences of the United States of America 28 (1942), 535-537.

[7] S. N. Mishra, Common fixed points of compatible mappings in PM-spaces, Mathematica Japonica 36 (1991), no. 2, 283-289.

[8] B. Schweizer and A. Sklar, Statistical metric spaces, Pacific Journal of Mathematics 10 (1960), 313-334. 
[9] V. M. Sehgal and A. T. Bharucha-Reid, Fixed points of contraction mappings on probabilistic metric spaces, Mathematical Systems Theory 6 (1972), no. 1-2, 97-102.

[10] S. L. Singh and B. D. Pant, Coincidence and fixed point theorems for a family of mappings of Menger spaces and extension to uniform spaces, Mathematica Japonica 33 (1988), no. 6, 957-973.

[11] Common fixed points of a family of mappings in Menger and uniform spaces, Rivista di Matematica della Università di Parma. Serie IV 14 (1988), 81-85 (1989).

[12] M. Stojaković, Common fixed point theorems in complete metric and probabilistic metric spaces, Bulletin of the Australian Mathematical Society 36 (1987), no. 1, 73-88.

Chi-Ming Chen: Department of Applied Mathematics, National Hsin-Chu University of Education, 300 Taiwan, China

E-mail address: ming@mail.nhcue.edu.tw

Tong-Huei Chang: Department of Applied Mathematics, National Hsin-Chu University of Education, 300 Taiwan, China

E-mail address: thchang@mail.nhcue.edu.tw 


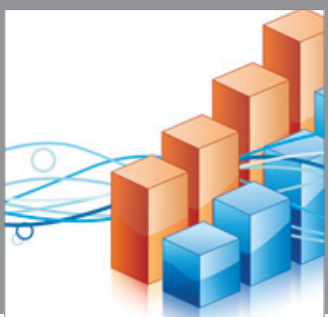

Advances in

Operations Research

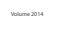

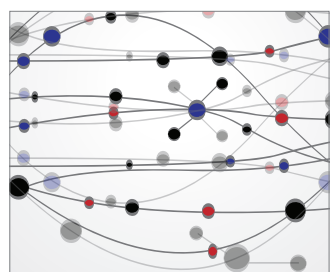

\section{The Scientific} World Journal
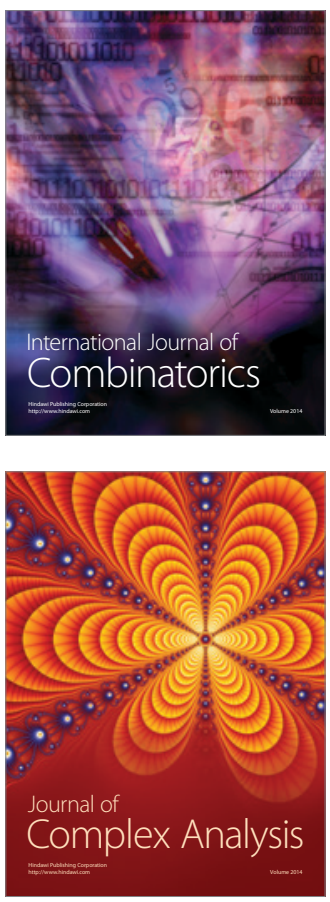

International Journal of

Mathematics and

Mathematical

Sciences
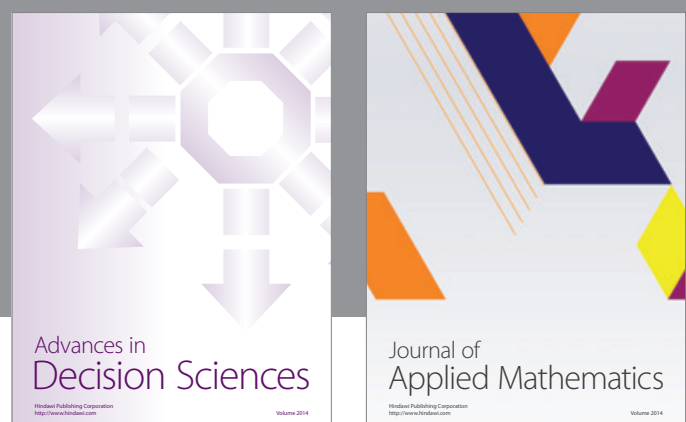

Journal of

Applied Mathematics
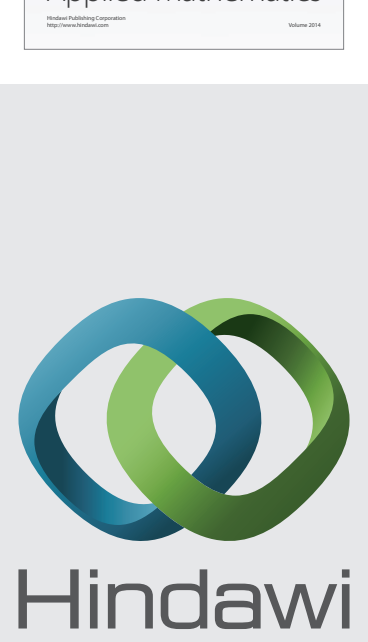

Submit your manuscripts at http://www.hindawi.com
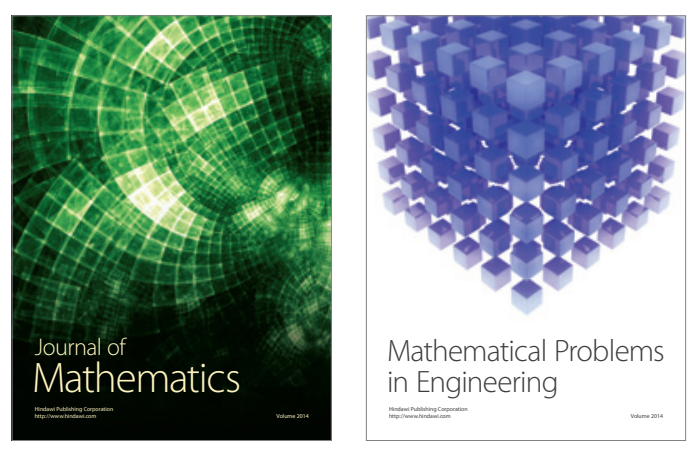

Mathematical Problems in Engineering
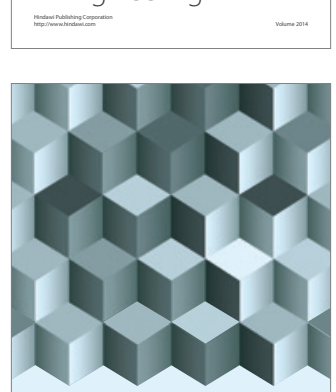

Journal of

Function Spaces
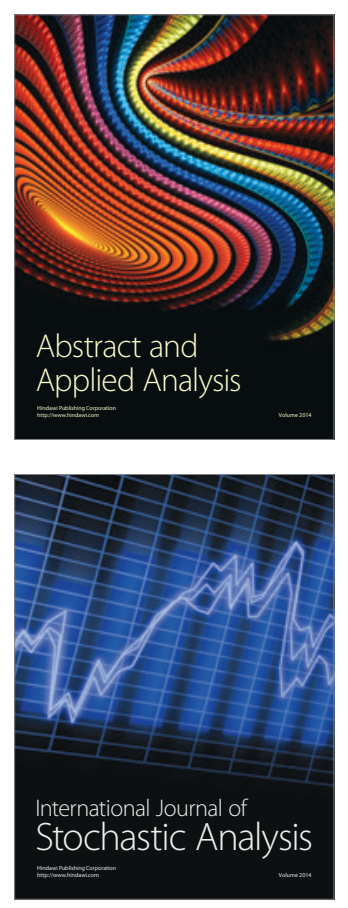

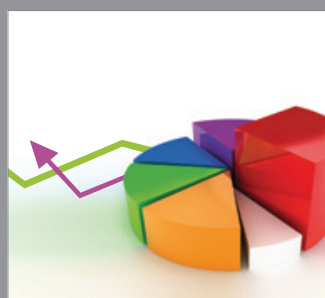

ournal of

Probability and Statistics

Promensencen
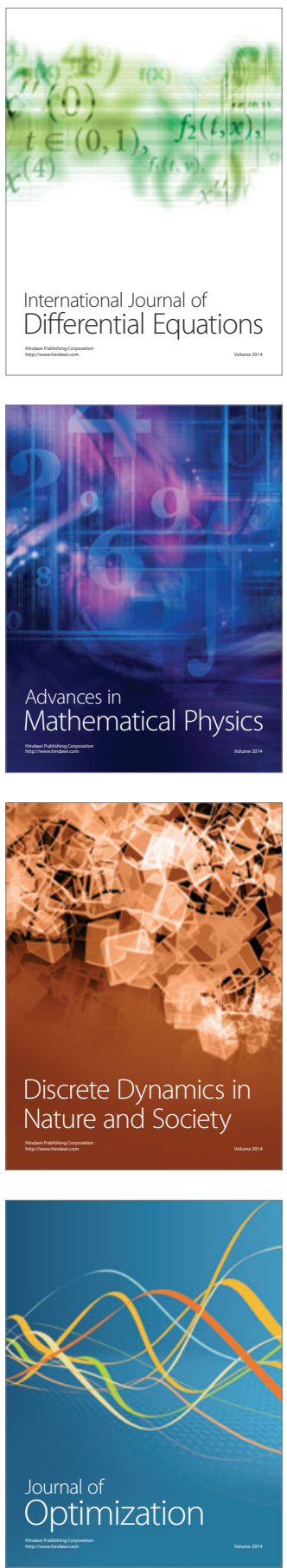